\title{
About Adjacency Matrix of Semigraphs
}

\author{
C. M. Deshpande and Y. S. Gaidhani
}

\begin{abstract}
The aim of the paper is to define the adjacency matrix of a Semigraph. We expect to see many applications of this in the future. Incidence matrix of a semigraph has been defined by the authors[4].Here we not only define Adjacency Matrix associated with a semigraph, but also state necessary and sufficient conditions for a matrix to be semigraphical. At the end of the paper spectrum of a semigraph is defined and some of its spectral properties are studied.
\end{abstract}

Index Terms-Adjacency matrix of a semigraph, spectrum of a semigraph.

\section{INTRODUCTION}

Semigraphs were introduced by E.Sampathkumar[2] as generalization of graphs. Many authors [2], [3], [4] have studied properties of semigraphs. Semigraphs prove to be a better model than graphs in all those applications where instead of two points to be connected by an edge we need to connect several points by an edge and the order in which they appear in the edge is of prime importance.

Unique representation of any discrete structure in matrix form is important for applications in computer science.

In Section 2 we give some preliminary definitions and in Section 3 we give main definition and necessary and sufficient conditions for a matrix to be adjacency matrix of a semigraph. Some spectral properties of adjacency matrix are discussed in Section 4.

\section{DEFINITIONS}

\section{Semigraph:}

A semigraph $G$ is a pair $(V, X)$ where $V$ is a non-empty set whose elements are called vertices of $G$ and $X$ is a set of $n$ tuples, called edges of $G$, of distinct vertices, for various $\mathrm{n}$ $(n \geq 2)$ satisfying the following conditions:

(i) Any two edges have at most one vertex in common

(ii) Two edges $\left(u_{1}, u_{2}, \ldots, u_{n}\right)$ and $\left(v_{1}, v_{2}, v_{m}\right)$ are considered to be equal if
(a) $\quad m=n$ and $\quad$ (b) either $u_{i}=v_{i}$ for $i=1,2, \ldots, n$ or $u_{i}=v_{n+1-i}$, for $i=1,2, \ldots, n$

For the edge $E=\left(u_{1}, u_{2}, \ldots u_{n}\right), u_{1}$ and $u_{n}$ are called the end vertices of $\mathrm{E}$ and $\mathrm{u}_{2}, \mathrm{u}_{3}, \ldots \mathrm{u}_{\mathrm{n}-1}$ are called the middle vertices of $E$.

\section{Adjacent vertices:}

Manuscript received April 14, 2012; revised May 26, 2012.

C. M. Deshpande is with the College of Engineering, Pune (e-mail: hod.maths@coep.ac.in)

Y. S. Gaidhani is with the M. E. S. Abasaheb Garware College, Pune. (e-mail: yogeshrigaidhani@gmail.com).
Two vertices in a semigraph are said to be adjacent if they belong to the same edge and are said to be consecutively adjacent if in addition they are consecutive in order as well For example, in the semigraph $G$ of Fig.1, the vertices $v_{1}$ and $v_{4}$ are adjacent while the vertices $v_{2}$ and $v_{3}$ are consecutively adjacent.

\section{Cardinality:}

Cardinality of an edge $E$ in a semigraph $G$ is the number of vertices lying on that edge. In the semigraph $G$ of Fig.1, cardinality of $E_{1}$, denoted as $\left|E_{1}\right|$, is 4 .

\section{Adjacent Edges:}

Two edges $E_{1}$ and $E_{2}$ in a semigraph $G$ are said to be adjacent if they have a vertex in common. For instance, in the semigraph $G$ of Fig.1, the edges $E_{2}$ and $\mathrm{E}_{3}$ are adjacent.

p-edge:

p-edge (partial edge) of $E$ is a $(k-j+1)$-tuple $E^{\prime \prime}=\left(v_{i j}\right.$, $\left.v_{i j+1}, \ldots, v_{i k}\right)$ where $1 \leq j<k \leq n$.

\section{$f$-edge:}

$f$-edge is any edge of a semigraph, called full edge.

fp-edge is an edge which is either an f-edge or a p-edge.

$A$ semigraph $G$ may be drawn as a set of points representing the vertices. An edge $E=\left(v_{i 1}, v_{i 2}, \ldots, v_{i r}\right)$ is represented by a Jordan curve joining the points corresponding to the vertices $v_{i 1}, v_{i 2}, \ldots, v_{i r}$ in the same order as they appear in $E$. The end points of the curve (i.e. the end vertices of $E$ ) are denoted by thick dots. The points lying on an edge in between the end points (i.e. middle vertices of $E$ ) are denoted by small hollow circles. If an end vertex $\mathrm{v}$ of an edge $\mathrm{E}$ is a middle vertex of some edge $E^{\prime}$, a small tangent is drawn to the circle (representing $v$ on $E^{\prime}$ ) at the end of E.

Fig. 1 represents the semigraph $G . E_{1}=\left(v_{1}, v_{2}, v_{3}, v_{4}\right), E_{2}$ $=\left(v_{1}, v_{6}, v_{5}\right), E_{3}=\left(v_{4}, v_{6}, v_{7}\right), E_{4}=\left(v_{4}, v_{5}\right), E_{5}=\left(v_{5}, v_{9}\right), E_{6}=$ $\left(v_{5}, v_{7}\right)$

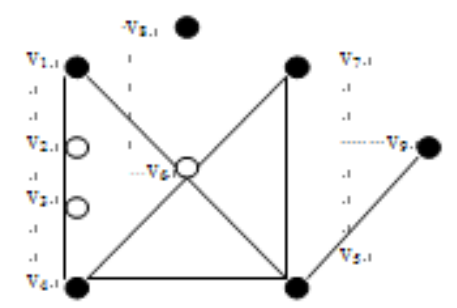

Fig. 1. Semigraph G.

\section{A. Remarks:}

- A semigraph with $n=2$ is a graph. Hence it can be seen as a natural generalization of graphs.

- A semigraph is a linear hypergraph $\mathrm{H}$ with an order given to each edge of $H$. The adjacency matrix associated with a semigraph as defined in this paper can shed more light on the properties of linear hypergraphs. 


\section{AdJACENCY MATRix OF A SEMIGRAPH}

Definition: Adjacency Matrix Associated with a Semigraph:

Let $G(V, X)$ be a semigraph with vertex set $V=\{1,2, \ldots, p\}$ and edge set $X=\left\{e_{1}, e_{2}, \ldots, e_{q}\right\}$ where

$e_{j}=\left(i_{1}, i_{2}, \ldots, i_{k j}\right), j=1,2 \ldots, q$ and $i_{1}, i_{2}, \ldots, i_{k j}$ are distinct elements of $\mathrm{V}$.

Adjacency matrix, $A$, of $G(V, X)$ is a $p \times p$ matrix whose entries are given by

$a_{i, j}=$ cardinaliy of fp-edge $\left(v_{i}, v_{j}\right)-1$; if $v_{i}, v_{j}$ are adjacent $=0$; otherwise.

As every $\mathrm{p}$-edge of cardinality $\geq 2$ belongs to exactly one f-edge of $\mathrm{G}$, the above matrix is well defined.

We label the rows and columns of $A$ as $1,2,3, \ldots, \mathrm{p}$; the same as vertex set of $G$.

Clearly, a row/column corresponding to an isolated vertex is a zero row/column. For the rest of the paper, without loss of generality, we consider semigraphs without isolated vertices.

We explain the definition with an example below and make some important observations.

Example 1: $G=(V, X)$ where $V=\{1,2,3,4,5,6,7,8\}$, $X=\left\{e_{1}(1,2,3,4,5,6), e_{2}(2,8), e_{3}(4,7)\right\}$.

$$
A=\left[\begin{array}{llllllll}
0 & 1 & 2 & 3 & 4 & 5 & 0 & 0 \\
1 & 0 & 1 & 2 & 3 & 4 & 0 & 1 \\
2 & 1 & 0 & 1 & 2 & 3 & 0 & 0 \\
3 & 2 & 1 & 0 & 1 & 2 & 1 & 0 \\
4 & 3 & 2 & 1 & 0 & 1 & 0 & 0 \\
5 & 4 & 3 & 2 & 1 & 0 & 0 & 0 \\
0 & 0 & 0 & 1 & 0 & 0 & 0 & 0 \\
0 & 1 & 0 & 0 & 0 & 0 & 0 & 0
\end{array}\right]
$$

We make the following observations:

- A is a real valued symmetric matrix, and $a_{i, i}=0 \forall i$

- $a_{i, j} \in\{0,1,2, \ldots, p-1\} \forall i, j$ where $\mathrm{p}$ is the number of vertices. Moreover, if there is an entry $p$ - 1 then the row and column containing that entry must have all the entries from 1 to $p$ - 1 . Also, in this case the semigraph will necessarily contain an edge of maximum length i.e.p.

- The sub matrices of A corresponding to edges $\mathrm{e}_{1}, \mathrm{e}_{2}$, $\mathrm{e}_{3}$ are respectively given by $\mathrm{A}_{1}, \mathrm{~A}_{2}, \mathrm{~A}_{3}$

$$
\text { where } \begin{aligned}
A_{1} & =\left[\begin{array}{llllll}
0 & 1 & 2 & 3 & 4 & 5 \\
1 & 0 & 1 & 2 & 3 & 4 \\
2 & 1 & 0 & 1 & 2 & 3 \\
3 & 2 & 1 & 0 & 1 & 2 \\
4 & 3 & 2 & 1 & 0 & 1 \\
5 & 4 & 3 & 2 & 1 & 0
\end{array}\right], \\
A_{2} & =\left[\begin{array}{ll}
0 & 1 \\
1 & 0
\end{array}\right],
\end{aligned}
$$

All the remaining entries of $A$ are 0 .

\section{Definition: Semigraphical Matrix:}

A $p \times p$ matrix $M$ is said to be semigraphical if there exists a semigraph $G$ on $p$ vertices having adjacency matrix as $M$.

\section{A. Necessary and Sufficient Conditions for a Matrix to Be} Semigraphical:

Theorem 1: $A \quad p \times p$ matrix $A=\left[a_{i j}\right]$ is the Adjacency matrix of a semigraph if and only if it satisfies following conditions:

- $a_{i, j}=a_{j, i}, \forall i, j$ and $a_{i, i}=0, \forall i$

- $a_{i, j} \in\{0,1,2, \ldots, p-1\} \quad \forall i, j$

- $\{1,2, \ldots, p\}$ can be expressed as a union of subsets $E_{1}$, $E_{2}, . ., E_{\mathrm{q}}$ such that $\left|E_{i}\right| \geq 2,|E i \cap E j| \leq 1 \forall i \neq j$ and if $E_{i}$ is a $(r+1)$ - subset containing $\left\{m_{0}, m_{1}, \ldots, m_{r}\right\} \subseteq$ $\{1,2, \ldots, p\}$ then $\mathrm{A}_{\mathrm{i}}$, the square sub matrix of $A$ of order $r+1$, obtained by considering $m_{0}, m_{1}, \ldots, m_{r}^{\text {th }}$ row and column entries of $\mathrm{A}$ is given by

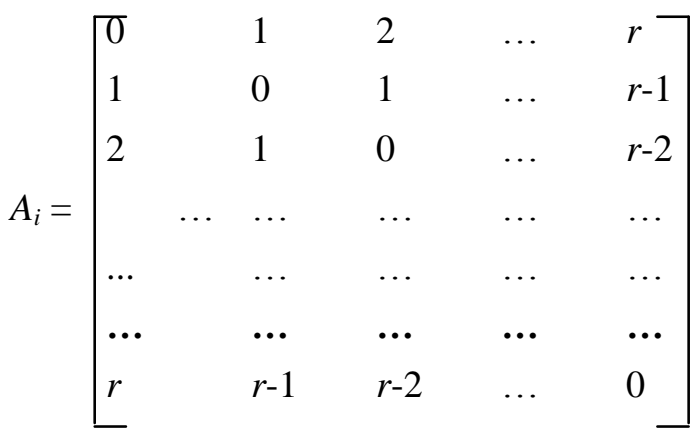

and all the remaining entries, if any, of $\mathrm{A}$ are 0 .

Proof : Suppose $G$ is a semigraph on p vertices $\{1,2, \ldots, p\}$ with adjacency matrix $A=\left[a_{i j}\right]$. By definition (i) and (ii) are satisfied. If $\left(m_{0}, m_{1}, m_{2}, \ldots, m_{r}\right)$ is an edge of $G$ then $m_{0}, m_{1}, m_{2}, \ldots, m_{r}^{\text {th }}$ row and column entries, by definition of adjacency matrix, are as given in $A_{i}$. Hence (iii) is satisfied. Conversely if (i), (ii),(iii) are satisfied then define a semigraph $G$ with vertex set $\{1,2, \ldots, p\}$ and edges as $E_{1}$, $E_{2}, . ., E_{q}$.

\section{Spectral Properties Of AdjaCenCy MatriX}

Let $\mathrm{A}$ be an adjacency matrix of a semigraph $G$. It follows from the definition of adjacency matrix that $A$ is a real, symmetric matrix. Suppose $\lambda$ is an eigen value of $A$. Then,

- since $A$ is real and symmetric, $\lambda$ is real.

- The multiplicity of $\lambda$ as a root of the characteristic equation det $(\lambda I-A)=0$ is equal to the dimension of the space of eigenvectors corresponding to $\lambda$.

\section{A. Definition: Spectrum of a Semigraph}

Definitions: Eigen values, Characteristic Polynomial of a semigraph:

Eigenvalues of the adjacency matrix, $A$, of a semigraph are called the eigenvalues of $\mathrm{G}$ and the characteristic polynomial of $A$ as the characteristic polynomial of $G$. Characteristic Polynomial of $G$ is denoted by $\chi(G, \lambda)$.

Let the characteristic polynomial of $\mathrm{G}$ be $\chi(G, \lambda)=\lambda^{p}+c_{1}$ $\lambda^{(p-1)}+c_{2} \lambda^{(p-2)}+\ldots+c_{p}$

The spectrum of a semigraph $G$ is the set of numbers which are eigenvalues of $\mathrm{G}$, together with their multiplicities.

If $\lambda_{0}>\lambda_{1}>\ldots>\lambda_{(s-1)}$ are distinct eigenvalues of $\mathrm{A}$ and their 
multiplicities are $\mathrm{m}_{0}, \mathrm{~m}_{1}, \ldots, \mathrm{m}_{(\mathrm{s}-1)}$ respectively, then

$$
\operatorname{spec}(G)=\left(\begin{array}{cccc}
\lambda_{0} & \lambda_{1} & \ldots & \lambda_{(s-1)} \\
m_{0} & m_{1} & \ldots & m_{(s-1)}
\end{array}\right)
$$

Example 3: Consider a semigraph given by the adjacency matrix, $A(G)=\left[\begin{array}{ccc}0 & 1 & 2 \\ 1 & 0 & 1 \\ 2 & 1 & 0\end{array}\right]$

Characteristic polynomial of $\mathrm{G}$ is $\lambda^{3}-6 \lambda-4=0$ and the eigenvalues are $\lambda_{1}=-2, \lambda_{2}=1+\sqrt{3}, \lambda_{3}=1-\sqrt{ } 3$

\section{Properties:}

As $\mathrm{A}$ is a real, symmetric matrix, trace of $A=0$.

As diagonal elements of A are all zero, coefficient of $\lambda^{(\mathrm{p}-1)}$ in $\chi(G, \lambda)$ is zero.

Remark: If $G_{1}$ and $G_{2}$ are isomorphic semigraphs then the adjacency matrix of one can be obtained by row and column permutations of the adjacency matrix of the other. Hence the two adjacency matrices are similar. Therefore $G_{1}, G_{2}$ have same spectrum.

Cospectral semigraphs: Two semigraphs are said to be cospectral if they have same spectrum.

Proposition: If $G_{1}$ and $G_{2}$ are isomorphic semigraphs then they are cospectral.

Proof of the proposition is obvious from the remark above.

Converse of the above statement is not true. In fact, we give below an example of two graphs that have the same spectrum but they are non isomorphic.

Example 4: Consider the graphs $G_{1}\left(V_{1}, E_{1}\right)$ and $G_{2}\left(V_{2}, E_{2}\right)$. $V_{1}=\{1,2,3,4,5,6\}, E_{1}=\{(1,2),(2,3),(2,4),(3,4),(3,5),(4,5),(5$, 6) $\}$,

$V_{1}=V_{2}, E_{2}=\{(1,2),(1,3),(2,3),(4,3),(5,3),(6,3),(5,6)\}$

Eigen values of $G_{1}$ and $G_{2}$ are same, but $G_{1}$ and $G_{2}$ are not isomorphic.

\section{REFERENCES}

[1] F. Harary, "Graph Theory," Narosa Publishing House, New Delhi, 1988.

[2] E. Sampathkumar, "Semigraphs and their applications," Report on the DST (Department of Science and Technoloy) project, submitted to DST, India, May 2000.

[3] N. S. Bhave, C. M. Deshpande, and B. Y. Bam, "Characterization of Potentially Hamiltonian graph in terms of Dual Semigraph," in Proceedings of 3rd National Conference, Mathematical Techniques: Emerging Paradigms for Electronics and IT Industries. Mateit-2010, vol. 4, pp. PS10.1-PS10, 2010

[4] C. M. Deshpande and Y. S. Gaidhani, "Matrix Representation of Semigraphs," 7th National Conference, Academy for Discrete Mathematics and Applications, NIT, Calicut, India. 